\title{
Discovery of metastable tetragonal disordered phase upon phase transitions in the equiatomic nanostructured FePd alloy
}

\author{
N.I. Vlasova ${ }^{\text {a,b,*}, ~ A . G . ~ P o p o v ~}{ }^{\text {a }}$, N.N. Shchegoleva ${ }^{\text {a,b }}$, V.S. Gaviko ${ }^{\text {a }}$, L.A. Stashkova ${ }^{a}$, \\ G.S. Kandaurova ${ }^{\mathrm{b}}$, D.V. Gunderov ${ }^{\mathrm{c}}$ \\ a Institute of Metal Physics, UD RAS, 18 S. Kovalevskoi Str., 620990 Ekaterinburg, Russia \\ ${ }^{\mathrm{b}}$ Ural Federal University, 19 Mira Str., 620002 Ekaterinburg, Russia \\ ${ }^{\mathrm{c}}$ Ufa State Avia-Technical University, 12 K. Marx Str., 450000 Ufa, Russia
}

Received 31 August 2012; received in revised form 18 January 2013; accepted 20 January 2013

Available online 20 February 2013

\begin{abstract}
Specific features of the phase transformation $\mathrm{A} 1 \rightarrow \mathrm{L}_{0}$ (space groups $F m \overline{3} m$ and $P 4 / m m m$, respectively) in single crystals of the equiatomic alloy FePd subjected to annealings both in the absence and in the presence of external uniaxial load, as well as in polycrystalline samples that had undergone severe plastic deformation via high-pressure torsion and subsequent annealing, have been studied. An investigation of the single crystals in a nanostructured state formed at different stages of ordering annealing was performed using optical polarization, thermomagnetic and transmission electron microscopy (TEM) methods. The nanostructured state of the polycrystalline samples FePd after deformation of both disordered and ordered FePd alloys and subsequent annealing was examined with the help of TEM and X-ray techniques. The results obtained were analyzed based on the known concepts of the symmetry theory of phase transitions. It was concluded that the atomic ordering in the FePd alloys is preceded by the formation of a ferroelastic disordered bodycentered tetragonal phase with the structural type A6 and the space group I4/mmm. Experimental data that evidence the existence of a tetragonal disordered phase both in the single crystals and in the polycrystalline samples of FePd after severe plastic deformation and subsequent annealing are reported. Thus, the $\mathrm{A} 1 \leftrightarrow \mathrm{A} 6 \leftrightarrow \mathrm{L} 1_{0}$ phase transformation represents a combination of different types of phase transitions such as cooperative displacement $\mathrm{A} 1 \leftrightarrow \mathrm{A} 6$ and ordering $\mathrm{A} 6 \leftrightarrow \mathrm{L} 1_{0}$ of atoms.
\end{abstract}

(c) 2013 Acta Materialia Inc. Published by Elsevier Ltd. All rights reserved.

Keywords: $\mathrm{L1}_{0}$ alloy FePd; Disorder-to-order, Order-to-disorder phase transformations; Martensitic transitions; TEM microscopy; X-ray diffraction

\section{Introduction}

The equiatomic FePd alloy in an atomically disordered state (just as the affined ferromagnetic alloys $\mathrm{Co}-\mathrm{Pt}, \mathrm{Fe}-$ $\mathrm{Pt}$, etc.) is conventionally believed to possess a face-centered cubic (fcc) lattice (A1 phase) and assigned to the space group $F m \overline{3} m$. After annealing at $T<T_{\text {cr }}\left(T_{\text {cr }}\right.$ is the critical temperature of the phase transition $\mathrm{A} 1 \rightarrow \mathrm{L}_{0}$ ) an ordered tetragonal $\mathrm{L1}_{0}$ phase with the space group

\footnotetext{
* Corresponding author at: Institute of Metal Physics, UD RAS, 18 S. Kovalevskoi Str., 620990 Ekaterinburg, Russia. Tel.: +7 34337837 82; fax: +7 3433745244 .

E-mail address: vlasova@imp.uran.ru (N.I. Vlasova).
}

$P 4 / m m m$ nucleates and grows in the alloy $[1,2]$. In the ordered or partially ordered states the alloys display a unique combination of high values of saturation magnetization $\left(\mu_{0} M_{\mathrm{s}}=1.0-1.37 \mathrm{~T}\right)$, constant of magnetocrystalline anisotropy $\left(\mathrm{K} \approx 10^{6}-10^{7} \mathrm{~J} \mathrm{~m}^{-1}\right)$, magneto-optical characteristics, deformation and corrosion-resistant properties. This combination offers the possibility of using the above alloys as thin-film media for high-density recording and storage of information or as permanent magnets for special purposes [3].

According to the modern first-principles theory of magnetic anisotropy [4-6], the magnetocrystalline anisotropy of $\mathrm{L}_{0}$ alloys is mainly related to the process of atomic ordering and depends on the degree of long order. It is 
experimentally shown that variation of temperature and duration of the ordering annealing and application of magnetic field or stresses in the course of this annealing, as well as of preliminary plastic deformation, are factors that exert a significant effect on the integral degree of long ordering, morphology and sizes of the meso- and microstructure elements of $\mathrm{L1}_{0}$ alloys [7-14]. In the majority of theoretical and experimental works (e.g. [4-15]) the tetragonal distortion of the crystal lattice, which arises in the alloys upon the phase transformation $\mathrm{A} 1 \rightarrow \mathrm{L1}_{0}$, is considered to result exclusively from layer-by-layer distribution of atoms of different kinds in the directions $\langle 100\rangle$ (Fig. 1).

However, in accordance with the symmetry theories of phase transformations [16-19], besides the transition $F m \overline{3} m \rightarrow P 4 / m m m$, the structural transitions $\mathrm{Fm} \overline{3} \mathrm{~m} \rightarrow I 4 / \mathrm{mmm}$ and $I 4 / \mathrm{mmm} \rightarrow P 4 / \mathrm{mmm}$ are also possible. Therefore, upon annealing of the alloys $\mathrm{Fe}-\mathrm{Pd}, \mathrm{Co}-\mathrm{Pt}$ and $\mathrm{Fe}-\mathrm{Pt}$ there can take place two possible scenarios of the phase transformation $\mathrm{A} 1 \rightarrow \mathrm{L}_{1}$ : (i) nucleation and growth of the $\mathrm{L}_{0}$ ordered domains $(F m \overline{3} m \rightarrow P 4 / \mathrm{mmm})$; (ii) displacive transformation of the fcc-phase into bodycentered tetragonal (bct)-phase and then transformation into $\mathrm{L}_{0}(\mathrm{Fm} \overline{3} \mathrm{~m} \rightarrow I 4 / \mathrm{mmm} \rightarrow P 4 / \mathrm{mmm})$. It is quite important to answer the question regarding the existence of a low-symmetry disordered bet phase. The answer to this question will contribute to a better understanding of not only the mechanisms responsible for the formation of magnetic hysteresis properties and features of magnetic phase transitions, but also the mechanisms by which the $\mathrm{A} 1 \leftrightarrow \mathrm{L1}_{0}$ phase transition occurs in the FePt, CoPt and FePd alloys. The first suppositions on the possibility of existence of a low-symmetry disordered phase in the CoPt alloy and a martensitic nature of its formation, which stems from the consideration of experimental results, were made in Ref. [20].

The appearance of a new phase upon the phase transformation is mostly clearly evidenced by observation of new reflections in the electron diffraction and X-ray patterns of the samples under study, compared to the starting material. Unfortunately, in the case under consideration there arise significant difficulties in the separation of the reflections of the new phase and matrix (especially at the earliest stages of transformation in the single crystals) because of the coherent coupling of the lattices and a certain relationship of the reflection sizes for two phases and the distance between them in the reciprocal lattice [21]. However, systematic optical polarization and transmission electron microscopy (TEM) studies of the single crystals FePd subjected to annealings both in the absence and in the presence of external uniaxial load have revealed a number of distinctive features of the behavior of meso- and microstructures upon the phase transformation $\mathrm{A} 1 \rightarrow \mathrm{L}_{0}$ [22-24]. In accordance with current concepts on martensitic [25-27] and, in particular, ferroelastic $[16,19,28-30]$ phase transitions, these distinctive features can be considered as the main signs of the formation of a ferroelastic tetragonal disordered phase upon the displacive (martensitic) phase transformation $\mathrm{Fm} \overline{3} m \rightarrow I 4 / \mathrm{mmm}(\mathrm{A} 1 \rightarrow \mathrm{A} 6)$.

It is known that in the single crystals and coarse polycrystals FePd the process of long atomic ordering proceeds rather slowly, even at optimal temperatures $\left(500-550{ }^{\circ} \mathrm{C}\right)$ [7,8,22-24]. The martensite phases proper rapidly form even at low temperatures and external stresses (elastic and plastic deformation) can favor the martensite transformation [31]. Therefore, it is reasonable to suppose that investigation of the phase transformation in the FePd alloys subjected to severe plastic deformation and subsequent annealing at sufficiently low temperatures can give additional information on the complex course of the phase transformation $\mathrm{A} 1 \rightarrow \mathrm{Ll}_{0}$. Detailed investigations of the phase transformation $\mathrm{A} 1 \rightarrow \mathrm{L1}_{0}$ in the polycrystalline samples of the equiatomic alloy $\mathrm{FePd}$ nanostructured via severe plastic deformation by high-pressure torsion (HPT) [32] and subjected to subsequent annealing were performed using TEM, X-ray analysis and magnetic measurements [33,34]. A comparative analysis of the results of combined study of the phase transformation $\mathrm{A} 1 \rightarrow \mathrm{L} 1_{0}$ in the single crystals and samples with an ultradisperse grain structure of the equiatomic alloy FePd made it possible to conclude on the formation of an intermediate bct

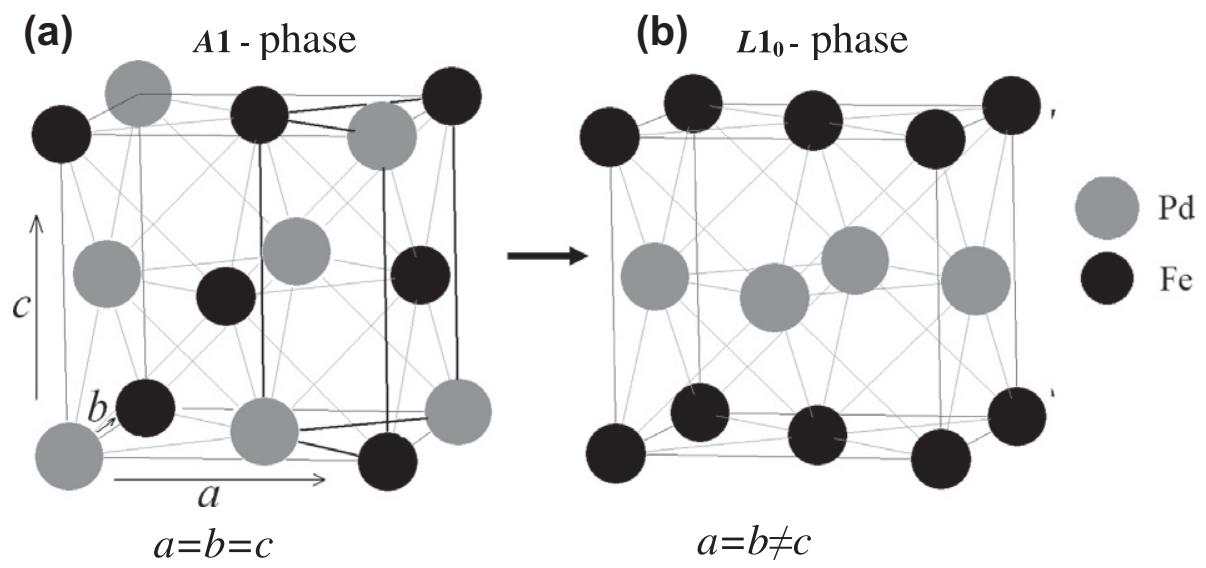

Fig. 1. Scheme of the phase transformation $\mathrm{Al} \rightarrow \mathrm{Ll}_{0}$ in the common representation $(\mathrm{fcc} \rightarrow \mathrm{fct}$ ). 
disordered phase A6 upon the phase transformation $\mathrm{A} 1 \rightarrow \mathrm{L} 1_{0}$. In the work presented, experimental evidence for the existence of an intermediate bct phase in single crystals and polycrystalline samples of the equiatomic alloy $\mathrm{FePd}$ is reviewed.

\section{Experimental}

Single crystals of the equiatomic alloy FePd were grown by the Bridgman method and oriented by the layer method. According to the X-ray data, after quenching to water from $T=950{ }^{\circ} \mathrm{C}$ the $\mathrm{FePd}$ single crystals were atomically disordered cubic single crystals with the lattice parameter $a_{\mathrm{fcc}}=0.3804 \mathrm{~nm}$. The optical polarization examination and thermomagnetic analysis were carried out on samples in the form of cubes or rectangular prisms whose faces were parallel to the crystallographic planes $\{100\}$ or $\{110\}$ of the starting cubic A1 single crystal. To reach the ordered state the single crystals of the FePd alloy were subjected to annealing at temperatures $T=500{ }^{\circ} \mathrm{C}, 550{ }^{\circ} \mathrm{C}$ and $600{ }^{\circ} \mathrm{C}$ without external actions or in the presence of uniaxial (tensile or compressive) load $\sigma=0.2 \mathrm{MPa}\left(2 \mathrm{~kg} \mathrm{~mm}^{-2}\right.$ ) at $T=550{ }^{\circ} \mathrm{C}$ and $600{ }^{\circ} \mathrm{C}$. It is known that, as the tetragonal $\mathbf{C}$ axis in the $\mathrm{L}_{0}$ lattice, any of the $\langle 100\rangle$ axes of the cubic A1 matrix may be selected. If annealing is performed in the absence of any external effects, the ordered phase contains crystalline $C$-domains of all three orientations $(\mathbf{C} 1, \mathbf{C} 2$ and $\mathbf{C} 3)$. Annealing under a uniaxial tensile or compressive load $\sigma \|\left[\begin{array}{lll}0 & 1\end{array}\right]_{\mathrm{A} 1}$ ensures the predominant growth of $C$-domains of two $(\mathbf{C} 1, \mathbf{C} 2)$ or one $(\mathbf{C} 3)$ orientations, respectively $[7,8,10,22]$.

Prior to quenching and after annealings for ordering the sample surfaces $\{100\}$ and $\{110\}$ were mechanically polished using diamond pastes with subsequently decreasing size of the abrasive particles from 10 to $0.1 \mu \mathrm{m}$ and/or electrolytically polished in concentrated hydrochloric acid. The crystal mesostructure and magnetic domain structure of the FePd crystals were observed using an optical polarization microscope (Neophot-2). The polycrystalline samples of the equiatomic FePd alloy in the atomically disordered and completely ordered states were subjected to severe plastic deformation by HPT at a pressure of $P=3$ and $6 \mathrm{GPa}$ with a different number $n$ of revolutions $(n=0,0.33,1.0$, 5 and 10) followed by annealings at $450{ }^{\circ} \mathrm{C}$. After the HPT deformation, there forms in the alloy a nanostructured state with a grain size $D=50-200 \mathrm{~nm}$. A short-term annealing at a rather low temperature $T=450{ }^{\circ} \mathrm{C}$ does not result in a significant increase of $D[33,34]$. The crystal structure and phase composition of the deformed and annealed samples were investigated with a DRON diffractometer using monochromatic $\mathrm{Cr} \mathrm{K} \alpha$ radiation. Calculation of the parameters of the crystal lattice was performed with the help of the program "Powder Cell" [35]. The microstructure of singlecrystal and polycrystalline samples FePd were examined using a JEM-200 CX transmission electron microscope. The temperature dependence of the initial magnetic susceptibility $\chi(T)$ in different crystallographic directions was measured on samples placed into quartz ampoules filled with helium in an AC magnetic field $H_{\mathrm{a}}=0.8 \mathrm{kA} \mathrm{m}^{-1}$ $(\sim 10 \mathrm{Oe})$ with a frequency of $80 \mathrm{~Hz}$. The designations of the crystallographic directions and planes used in the patterns are shown in the axes of the fcc lattice of the initial disordered phase.

\section{Results and discussion}

\subsection{The main signs of the formation of the tetragonal disordered phase upon the phase transformation $A 1 \rightarrow L 1_{0}$ in single crystals FePd}

\subsubsection{Formation of pre-transition state}

Some peculiar features of the FePd crystals are apparent immediately after heating to $T>T_{\mathrm{cr}}$ and quenching in water, namely the occurrence of a tweed contrast in the electron microscopy images and regions of diffuse
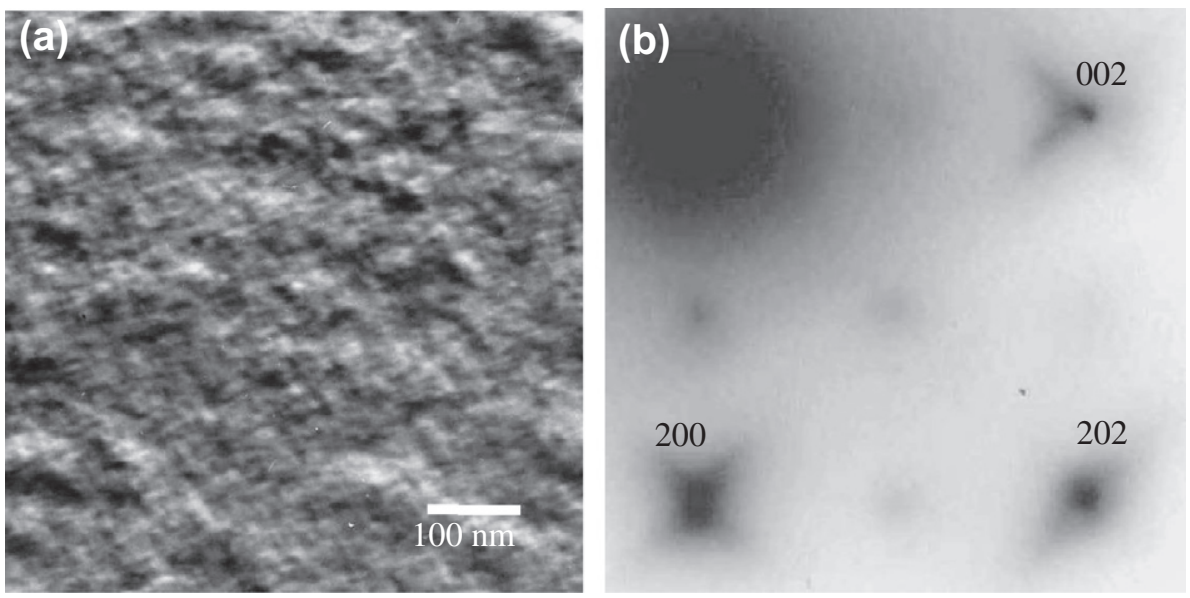

Fig. 2. (a) Light-field electron microscopy image and (b) electron diffraction pattern for the FePd crystal after annealing at $T=600{ }^{\circ} \mathrm{C}$ for $4 \mathrm{~h}$. The zone axis is close to [001]. 
scattering (RDS) that exist near the fundamental reflections in the electron diffraction patterns. These features become more pronounced after the annealing at temperatures of $500-600^{\circ} \mathrm{C}$. As an example, Fig. 2 shows a light-field electron microscopy image (Fig. 2a) and electron diffraction pattern (Fig. 2b) for the FePd crystal after annealing at $T=600^{\circ} \mathrm{C}$ for $4 \mathrm{~h}$. It must be emphasized that the tweed contrast and RDS near the fundamental reflections (002), (202) and (200) take place in the absence of superlattice reflections. One can see diffuse spots instead of the superlattice reflections. The RDS look like streaks oriented along the $\langle 110\rangle$ directions, and in their appearance, heavily resemble the RDS which were observed in disordered $\mathrm{Fe}-\mathrm{Ni}$ alloys in the vicinity of the martensitic $\gamma \rightarrow \alpha$ transformation point [36]. Similar patterns of the tweed microstructure and electron diffraction were also obtained at an early stage of the fcc-fct martensitic transformation in $\mathrm{Fe}-\mathrm{Pd}$ alloys containing 30 at.\% Pd [37].

The above peculiarities are the main signs of the formation in the material of a pre-transition state. The pretransition states are considered precursors of spontaneous martensitic transitions (including ferroelastic) which are realized via cooperative elastic displacements of atoms over the systems $\{110\}\langle 1 \overline{1} 0\rangle[27,29,30]$. The waves of elastic displacements of atoms periodically distort (modulate) the initial crystal lattice. In the frame of theoretical analysis of the martensitic transformations in the models of static atomic displacements, the RDS are considered to be analogs of the Bragg reflections of a new phase in the classical theory of heterogeneous fluctuations [38].

\subsubsection{Existence of macroscopic ferroelastic domains at earliest stages of ordering annealing}

As mentioned above, in FePd single crystals and coarse polycrystals the process of long-range ordering proceeds rather slowly, even at optimal temperatures. On the other hand, optical polarization studies have shown (see previously published data [22-24]) that even during the earliest stages of the ordering annealing $(15-30 \mathrm{~min}$ at $T=500{ }^{\circ} \mathrm{C}$ ) the $\mathrm{FePd}$ crystal contains macroscopic ferroelastic domains (FEDs) which have differently oriented optical axes. A pattern of optical polarization contrast observed on the surface $(001)$ in the FePd crystal after

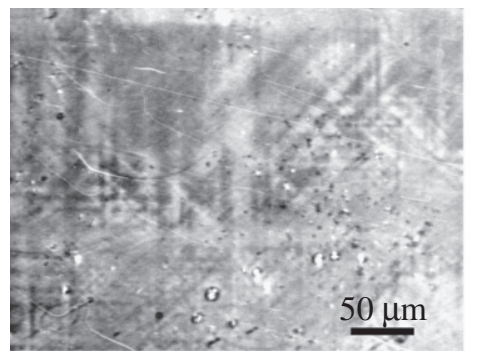

(001)

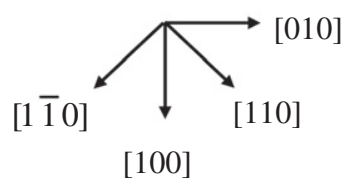

Fig. 3. Pattern of optical polarization contrast observed on surface $\left\{\begin{array}{ll}0 & 01\}\end{array}\right\}$ of the FePd crystal after annealing at $T=500{ }^{\circ} \mathrm{C}$ for $30 \mathrm{~min}$. annealing at $T=500{ }^{\circ} \mathrm{C}$ for $30 \mathrm{~min}$ is shown in Fig. 3. Since the fcc structure is optically isotropic, the fact of observance of the FEDs testifies to a lowered symmetry of the FePd crystal lattice. From the changes of the optical polarization contrast upon rotation of the FePd sample around the optical axis of the microscope it follows that the crystal lattice becomes tetragonal: optical axes in the adjacent FEDs are aligned with the axes $\langle 100\rangle$ and are misoriented with respect to each other by an angle close to $\pi / 2$, as in a tetragonally ordered crystal [39]. According to the electron microscopy data, the ordered phase is contained in the crystal in the form of optically unresolved nanoscaled regions $[8,23,24]$. Judging by the intensity relationship $I_{\mathrm{s}} / I_{\mathrm{f}}=0.01$ of the X-ray superlattice (sl) and fundamental (f) reflections after annealing of the equiatomic FePd alloy at $T=500{ }^{\circ} \mathrm{C}$ for $t=30 \mathrm{~min}$ [7], the volume fraction of the ordered phase is too low to be discerned by the optical polarization method [40]. Therefore, the observed macroscopic FEDs cannot be considered as domains of the ordered tetragonal phase. It should be assumed that here we deal with another tetragonal phase which, most likely, is disordered and serves as a matrix for ordered regions that arise.

\subsubsection{Occurrence of shear twins on surfaces coinciding with planes $\{111\}$ of the fcc lattice}

In the process of long annealings (100-200 h) of the FePd crystal at $T=\left(500-550^{\circ} \mathrm{C}\right)$ the structure of FEDs passes into a single-domain state (see previously published data [22-24]). If the annealing is performed in the presence of a uniaxial load, the single-domain state in ferroelastic crystal FePd is formed for several minutes. Either way, upon further annealing in the macroscopic single-domain portions of the FePd crystal, twins form (supposedly as a result of shear plastic deformation). From the optical polarization observation $[22,24]$ it follows that the traces of the above-mentioned twins on the surfaces $\{100\}$ coincide with directions close to $\langle 110\rangle$ of the starting fcc crystal, whereas on the surfaces $\{10 \overline{1}\}$, the traces of these twins close to the directions $\langle 101\rangle$ and $\langle 121\rangle$ are observed. This indicates that the twins conjugate in planes close in orientation to the $\{111\}$ planes of the initial fcc lattice. On the other hand, it is this set of traces $(\langle 101\rangle$ and $\langle 121\rangle)$ that can belong to the conjugation planes $\{101\}$ of the shear twins of the tetragonal body-centered phase which arises upon the martensitic phase transition $\mathrm{fcc} \rightarrow$ bct $(c / a \neq \sqrt{ } 2)$. (It is known that the most energetically favorable planes of conjugation of elastic domains and shear twins in a tetragonal crystal are $\{101\}$ planes $[21,41]$.) For instance, in the case of the Bain orientation relationship between the fcc and bct phases the planes $\{111\}$ of the initial phase become close the planes $\{101\}$ of the new bct phase, along which there can take place mechanical twinning. The TEM observations confirm the existence of twins with the conjugation planes parallel to the $\left\{\begin{array}{lll}1 & 1 & 1\end{array}\right\}$ planes of the fcc lattice in the FePd crystal subjected to annealings (Fig. 4). 

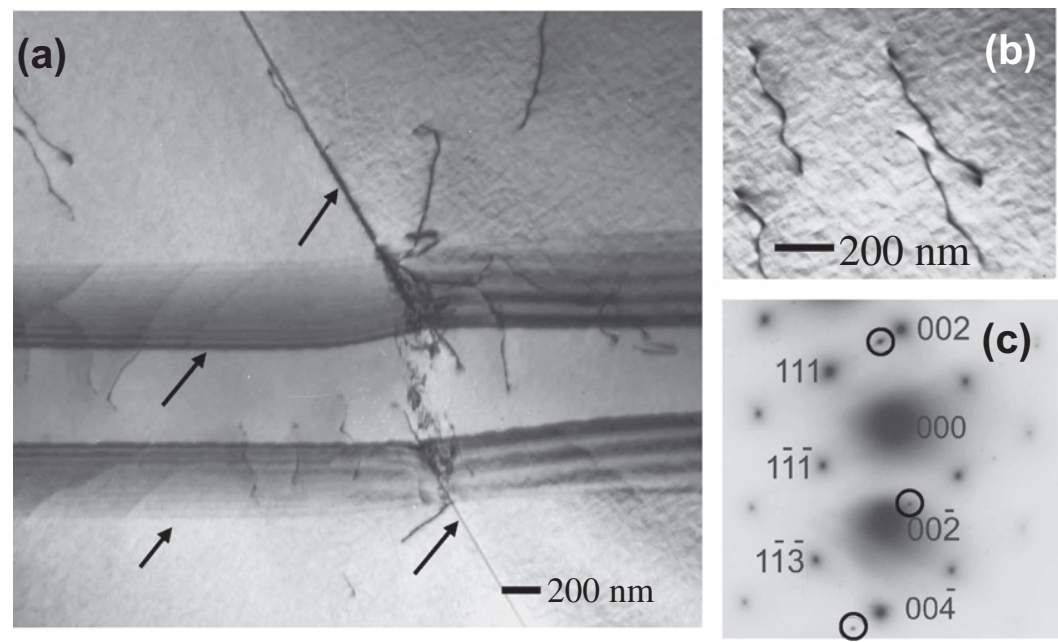

Fig. 4. (a) Twins oriented along the planes $\{111\}_{\text {fcc }}$ after annealing of the FePd crystal at $T=550{ }^{\circ} \mathrm{C}$ for 90 min in the presence of a tensile stress of $\sigma=0.2 \mathrm{MPa}$. (b) Magnified image of the tweed-like microstructure is shown. (c) The diffraction pattern related to the image in Fig. 4a. The zone axis is [1 110$]$. The twin reflections are enclosed in circles.

3.1.4. Coexistence of the tweed-like and antiphase contrasts

In the TEM images of the FePd crystals subjected to annealings for ordering both in the absence and in the presence of external uniaxial load the coexistence of the tweedlike and antiphase contrasts in one and the same domain of the sample can be observed (see previously published data $[23,24])$. An an example, Fig. 5a shows an image of the FePd crystal in the superlattice reflection (201) after annealing at $T=550^{\circ} \mathrm{C}$ for $1.5 \mathrm{~h}$ in the presence of a uniaxial load. The electron diffraction pattern from such an area selected inside the domain is shown in Fig. 5b. The contrast is seen to resemble that from the thermal antiphase boundaries (APBs). In the completely ordered material, the antiphase domains must look homogeneous in the crystal structure. However, in the fundamental reflection (002) one can see at the same spot a highly disperse tweed-like structure analogous to the structure shown in Fig. 4b. A weak tweed-like contrast inside the antiphase domains can be observed, even in the superlattice reflection (Fig. 5a). Therefore, an assumption can be made that the tetragonal crystal FePd is two-phase. Upon long-term annealings, traces of the antiphase domain boundaries on the surfaces $\{001\}$ tend to align with the directions $\langle\overline{1} 10\rangle$ и $\langle 100\rangle$. Hence, most probably, the antiphase boundaries are oriented along the planes $\{110\}[23,24]$. This contradicts a conventional opinion that the thermal APB in the $\mathrm{L}_{0}$ alloys are oriented along the planes (100) and (010) [42]. Note that the cooperative displacement of atoms upon the phase transformation $\mathrm{fcc} \rightarrow$ bct is realized through the systems $\{110\}\langle\overline{1} 10\rangle$ [27]. Feasibly, the origination and orientation of the "antiphase" boundaries observed are traceable to the $\mathrm{fcc} \rightarrow$ bct transition. To correctly interpret the above peculiarities of these boundaries, additional HRTEM investigations are needed.

\subsubsection{Transformation of magnetic domain structure after long-term annealing}

As the regions with a preferred orientation of the tetragonal axis $c$ become rather coarse $(t>50 \mathrm{~h})$, the magnetic domain structure (MDS) can be revealed using the polar
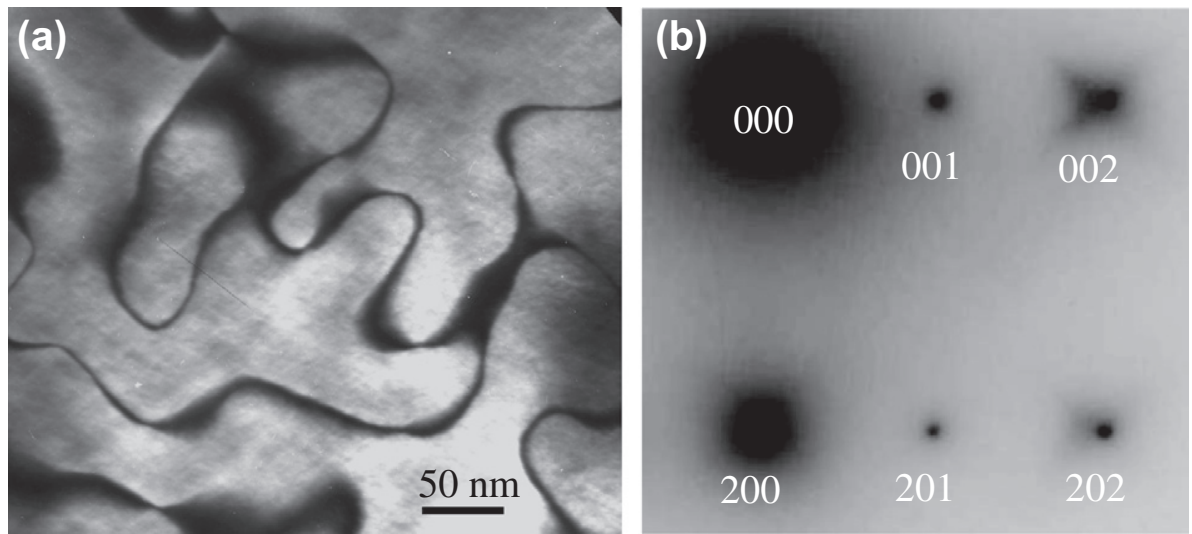

Fig. 5. (a) Electron microscopy image in the superlattice reflection (201) and (b) electron diffraction pattern for single-crystal FePd after annealing at $T=550^{\circ} \mathrm{C}$ for $90 \mathrm{~min}$ in the presence of a uniaxial tensile stress of $\sigma=0.2 \mathrm{MPa}$. Inside the antiphase domains there is observed a weak tweed-like contrast (compare with Fig. 4b). 
magneto-optical Kerr effect. It is worth noting that the MDS on the basis plane (001) of the tetragonal crystal FePd was not revealed by this method. At the same time, on a large portion of the $(10 \overline{1})$ surface tilted with respect to the tetragonal axis $c$ by the angle $45^{\circ}$, a complex star-like MDS (Fig. 6a) is observed that is rather typical of the basis plane of a massive magnetically uniaxial crystal [43]. If the easy magnetization axis (EMA), which is a tetragonal $c$ axis, is tilted toward the observation plane by an angle close to $\pi / 4$, the MDS of a homogeneous highly anisotropic magnetically uniaxial crystal must exhibit a picture of stripe-like domains oriented along the EMA projection onto this surface [44]. The stripe MDS (Fig. 6b) starts forming only after a long-term annealing of the FePd crystal. Such transformation of the configuration of the MDS may well arise as a result of changes in the amount and type of the effective magnetic anisotropy upon variations in the volume fractions of a disordered and an ordered tetragonal phases in the course of annealing.

\subsubsection{Existence of three critical temperatures in the dependences $\chi(T)$}

Finally, as a result of thermomagnetic studies of the crystals of equiatomic FePd alloy, the existence of three critical temperatures has been established: $T_{C 1} \approx 450{ }^{\circ} \mathrm{C}$, $T_{C 2} \approx 465^{\circ} \mathrm{C}$ and $T_{C 3} \approx 480-485^{\circ} \mathrm{C}$ (Fig. 7) at which there is observed a kink or maximum in the temperature dependences of the initial susceptibility $\chi(T)$ [23]. The temperatures $T_{C 1}$ and $T_{C 3}$ are the Curie temperatures of the fcc and $\mathrm{L1}_{0}$ phases of the FePd alloy, respectively [23,45,47]. Taking into account the total set of the above data, the

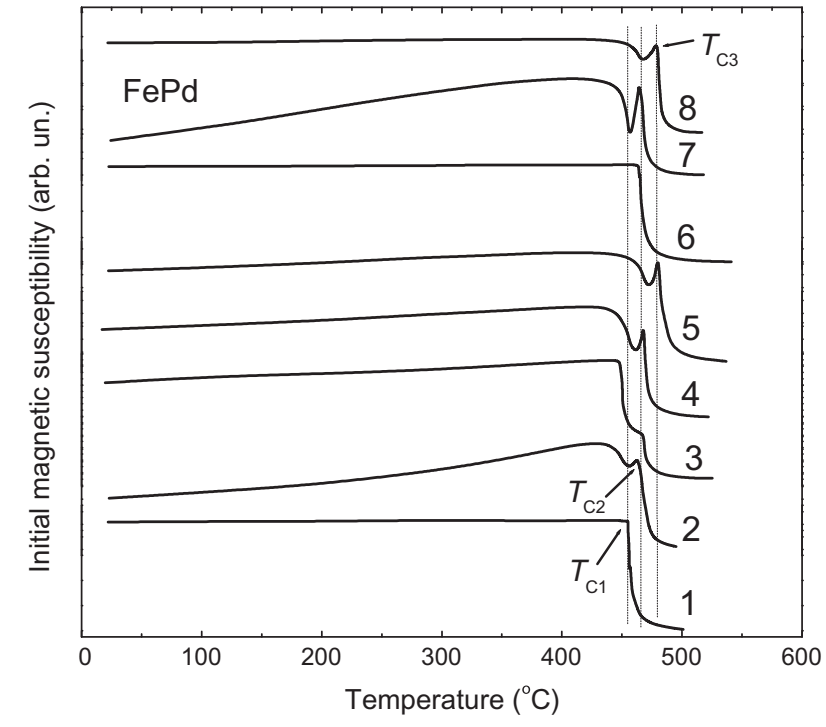

Fig. 7. Dependences $\chi(T)$ for the crystals FePd after (1) quenching from $950{ }^{\circ} \mathrm{C}$ and annealings at (2) $T=500^{\circ} \mathrm{C}$ for $180 \mathrm{~h}$; (3) $T=550^{\circ} \mathrm{C}, 12 \mathrm{~h}$; (4) $T=550{ }^{\circ} \mathrm{C}, 40 \mathrm{~h}$; (5) $T=550{ }^{\circ} \mathrm{C}, 100 \mathrm{~h}$ and (6) $T=600{ }^{\circ} \mathrm{C}, 100 \mathrm{~h}$ without loading; after (7) annealing at $T=600^{\circ} \mathrm{C}, 10 \mathrm{~min}$ and $\sigma=0.2 \mathrm{MPa}$ and (8) subsequent annealing of this sample without loading for $60 \mathrm{~h}$. Curves $\chi(T)$ are measured along easy magnetization axis. The positions of temperatures $T_{C 1}, T_{C 2}, T_{C 3}$ are marked by vertical dashed lines.

critical temperature $T_{C 2} \approx 465^{\circ} \mathrm{C}$ should evidently be taken as the Curie temperature of the tetragonal bct phase. The fact that the above peculiarities (existence of RDS in the vicinity of fundamental reflections in the electron diffraction patterns, coexistence of the tweed and antiphase
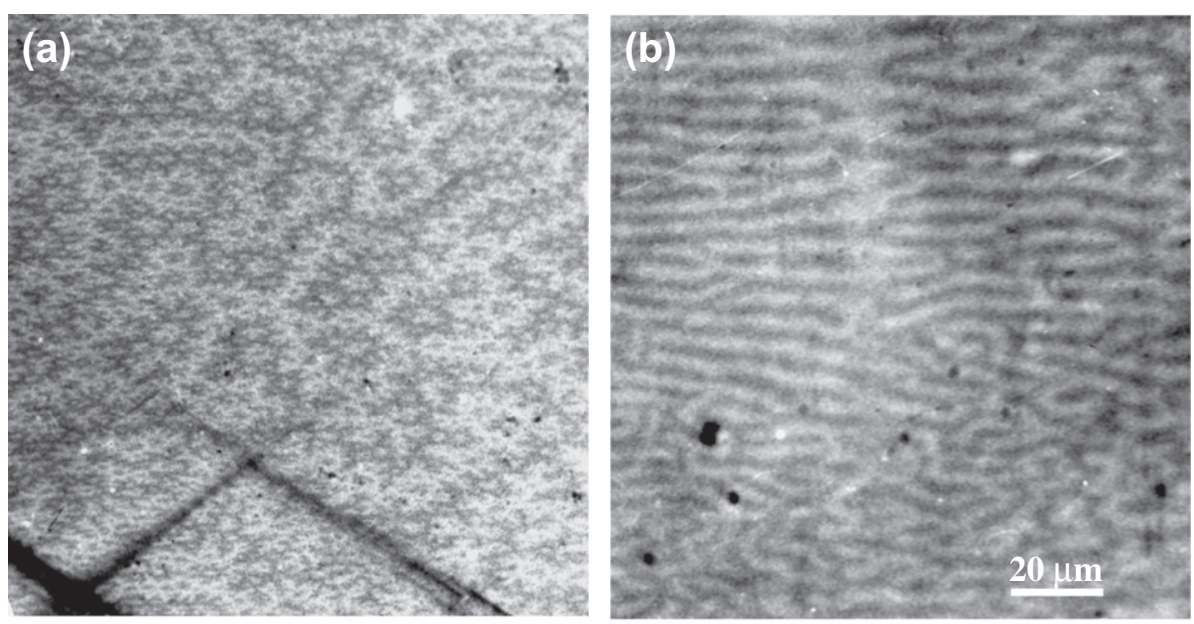

$(10 \overline{1})$

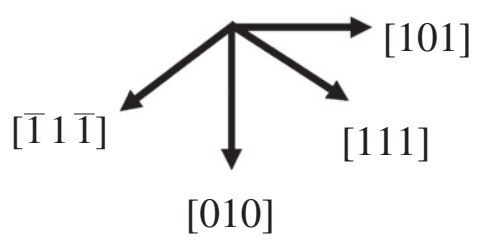

Fig. 6. Configuration of magnetic domains on one and the same surface $(10 \overline{1})$ of the FePd crystal after annealing at $T=500{ }^{\circ} \mathrm{C}$ for (a) $t=50 \mathrm{~h}$ and (b) $t=100 \mathrm{~h}$. 
contrasts in the TEM images, changes in the configuration of the MDS on one and the same surface $\{101\}$, anomalous behavior of the $\chi(T)$ curves near the $T_{C 2} \approx 465^{\circ} \mathrm{C}$ ) are retained after annealing for $100 \mathrm{~h}$ indicates an incompleteness of the process of ordering and the presence of the bct phase in the FePd single crystals. The existence of the tetragonal disordered phase is likely to cause remarkable differences in the Curie temperatures of the $\mathrm{L}_{0}$ phase in the FePd alloy, measured by different authors $[10,23,45-47]$.

\subsection{Experimental evidence of the existence of the tetragonal disordered phase in the equiatomic FePd alloy subjected to HPT deformation}

\subsubsection{Asymmetry and broadening of X-ray peaks}

A typical pattern of the X-ray diffraction for the HPT deformed samples of the disordered alloy FePd is shown in Fig. 8. It is seen that the fundamental reflections undergo a strong asymmetric broadening. The asymmetry broadening of the X-ray peaks can be traceable to overlapping of the closely located reflections of several phases. In our case, these are two disordered phases because no superlattice reflections are present in the X-ray pattern (the full pattern of the X-ray diffraction is shown in the inset of Fig. 8). It is natural to expect that one of them is the cubic A1 phase with the space group $F m \overline{3} m$. The analysis of the X-ray diffraction patterns using the program [35] shows that the second disordered phase is a bct phase with the space group $I 4 / \mathrm{mmm}$ and the structure type A6. Interpretation of the experimental X-ray pattern is shown in Fig. 8. The tetragonal crystal system (syngony [18]) is known to include only two types of the Bravais lattices: bet $(I)$ and primitive $(P)$ $[18,48]$. The latter reflects the spatial symmetry of the

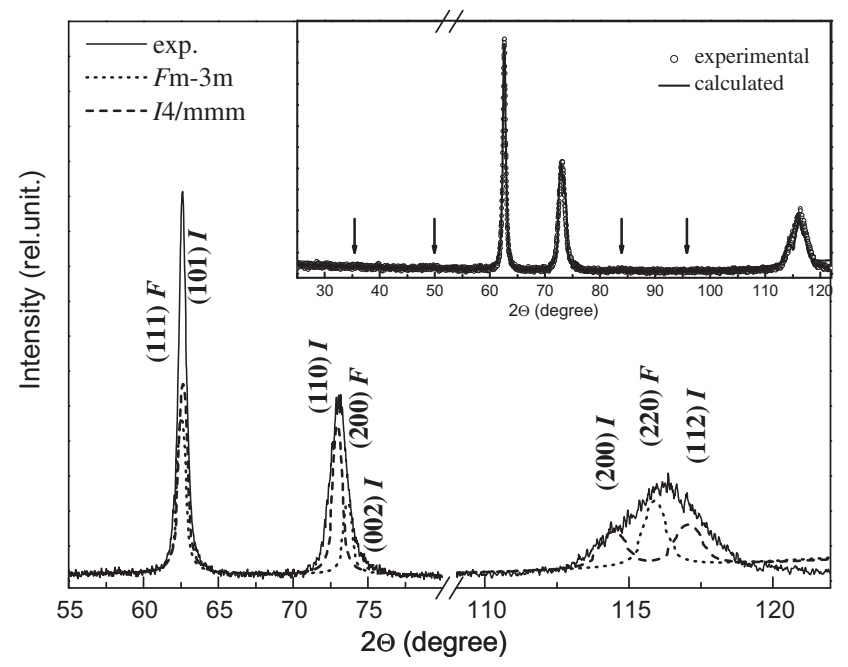

Fig. 8. Interpretation of the X-ray diffraction pattern for the FePd sample subjected to severe plastic deformation $(P=6 \mathrm{GPa}, n=5)$ in the disordered state. The inset shows the full X-ray diffraction pattern of the sample: experimental (line) and calculated (points). The arrows indicate positions of the superlattice reflections in the X-ray diffraction pattern for the ordered FePd alloy. ordered $\mathrm{L} 1_{0}$ phase. In the above $I$-representation the new tetragonal (bct) phase possesses the lattice parameters $a=0.2709 \mathrm{~nm}, c=0.3773 \mathrm{~nm}$ and features the ratio $c / a=1.393$. In the conventional fct representation this ratio is equal to $c / a=0.985$ with the given parameters $a=0.3831 \mathrm{~nm}, c=0.3773 \mathrm{~nm}$. According to the X-ray data, the fcc lattice has the parameter $a=0.3805 \mathrm{~nm}$.

\subsubsection{Tetragonal splitting of diffraction rings in the electron diffraction patterns of the HPT deformed FePd alloy}

The electron microscopy examination of the FePd samples does not allow us to perform a detailed analysis of microstructure because of rather fine grains and strainstressed state; yet electron diffraction patterns bear quite important information. Compare the electron diffraction patterns taken from two samples of the FePd alloy after different treatments, which are equally scaled in Fig. 9. The ring electron diffraction pattern of the FePd alloy subjected to HPT deformation ( $P=6 \mathrm{GPa}, n=5)$ in the disordered state is shown in Fig 9a. Fig. 9b shows the electron diffraction pattern taken after annealing of the HPT deformed FePd sample at $T=450^{\circ} \mathrm{C}$ for $11 \mathrm{~h}$. To start with, let us consider the electron diffraction pattern shown in Fig. 9b. It completely corresponds to that for the ordered $\mathrm{L}_{0}$ phase, regarding all fundamental (111), (200), (002), (220), (202), (311), (113), (222) and superlattice (001), (110), (201), (112), (221), (310) reflections. Splitting of the fundamental diffraction rings marked by pairs of two-pike arrows is traceable to the tetragonal distortion of the lattice. The position of superlattice

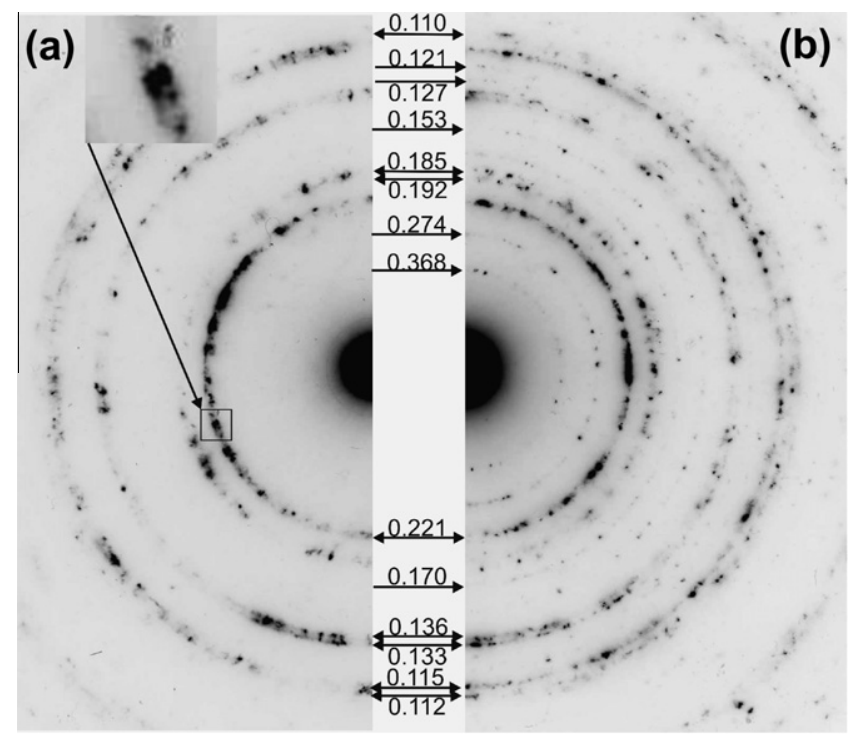

Fig. 9. Electron diffraction patterns of the samples (a) fabricated from the disordered equiatomic FePd alloy after severe plastic deformation ( $P=6 \mathrm{GPa}, \quad n=5$ revolutions) and (b) subsequent annealing at $T=450{ }^{\circ} \mathrm{C}$ for $11 \mathrm{~h}$. Two-pike arrows point to the positions of diffraction rings that are common for both electron diffraction patterns. One-pike arrows show the positions of the diffraction rings, those present only in the electron diffraction pattern (b). The values of interlattice spacings are shown above and below the arrows. In the inset (a) the magnified image of the portion of the split ring (111) is shown. 
reflections is indicated by one-pike arrows. The diffraction pattern in Fig. 9a is characterized by a similar splitting, in terms of position and magnitude, of the diffraction rings, with the superlattice reflections being absent. Thus, a direct comparison of two electron diffraction patterns in Fig. 9 allows a conclusion to be made that the left part of the figure shows the electron diffraction pattern of a disordered tetragonal crystal. The observed splitting of the (111) reflection ring (see the inset in Fig. 9a) indicates, according to estimates [49], that the samples contain many $\{111\}$ microtwins. At the same time, the splitting of the fundamental reflections does not exclude the presence of a cubic disordered phase in the samples under study, since it is difficult to discern reflections (200) and (220) from the fcc and fet phases.

To quantitatively describe the above electron diffraction patterns, in Table 1 there are shown calculated and experimental values of interlattice spacings $d$ for a possible set of crystal lattices in the samples FePd. Comparison of calculated and experimental values $d$, within the accuracy of the measurements of interlattice spacings in the electron diffraction patterns, evidences the presence of the tetragonal disordered phase in the HPT deformed alloy and formation of the ordered phase after annealing of the deformed samples FePd (compare columns $d$ : fct, dis; fct, ord and the corresponding italicized ones).

\subsubsection{Changes of the volume fractions of the ordered and} disordered phases upon the HPT deformation of the ordered $\mathrm{FePd}$ alloy and subsequent annealing

The results of X-ray studies of the equiatomic FePd alloy subjected to HPT deformation in the ordered state and subsequent annealing also allow a conclusion to be

Table 1

hkl Indexes and the corresponding values of the interlattice spacings for the cubic ( $\mathrm{fcc}$ ) phase and tetragonal disordered (fct, dis) and $\mathrm{L1}_{0}$ ordered (fct, ord) phases in the fct presentation. The values in normal type denote the spacings calculated from the lattice parameters $a$ and $c$, according to the X-ray data. The italicized $d$ values are gained from the electron diffraction patterns shown in Fig. 9. Calculation of the interlattice spacings was performed with allowance for the position of the ring (111), with the value $d_{111}=0.221 \mathrm{~nm}$ taken as a reference one.

\begin{tabular}{|c|c|c|c|c|c|}
\hline \multirow[t]{2}{*}{ hkl } & \multicolumn{3}{|c|}{ Calculation } & \multicolumn{2}{|c|}{ Experiment } \\
\hline & $\begin{array}{l}d(\mathrm{~nm}) \\
\mathrm{fcc}\end{array}$ & $\begin{array}{l}d(\mathrm{~nm}) \\
\mathrm{fct}, \mathrm{dis}\end{array}$ & $\begin{array}{l}d(\mathrm{~nm}) \\
\text { fct, ord }\end{array}$ & $\begin{array}{l}d(\mathrm{~nm}) \\
\text { fct, dis }\end{array}$ & $\begin{array}{l}d(\mathrm{~nm}) \\
\text { fct, ord }\end{array}$ \\
\hline 001 & - & - & 0.3684 & - & 0.3684 \\
\hline 110 & - & - & 0.2735 & - & 0.274 \\
\hline 111 & 0.2197 & 0.2200 & 0.2196 & 0.2197 & 0.221 \\
\hline 200 & 0.19025 & 0.1915 & 0.1934 & 0.192 & 0.192 \\
\hline 002 & - & 0.887 & 0.1842 & 0.187 & 0.185 \\
\hline 201 & - & - & 0.1712 & - & 0.170 \\
\hline 112 & - & - & 0.1528 & - & 0.153 \\
\hline 220 & 0.1345 & 0.1354 & 0.1367 & 0.136 & 0.136 \\
\hline 202 & - & 0.1344 & 0.1334 & 0.133 & 0.133 \\
\hline 221 & - & - & 0.1280 & - & 0.127 \\
\hline 310 & - & - & 0.1220 & - & 0.121 \\
\hline 311 & 0.1147 & 0.1160 & 0.1160 & 0.115 & 0.115 \\
\hline 113 & - & 0.1120 & 0.1110 & 0.112 & 0.112 \\
\hline 222 & 0.1097 & 0.1100 & 0.1100 & 0.110 & 0.110 \\
\hline
\end{tabular}

made that upon the phase transformations $\mathrm{L}_{0} \rightarrow \mathrm{A} 1$ and $\mathrm{A} 1 \rightarrow \mathrm{L1}_{0}$ a low-symmetry disordered phase is formed [33]. After annealing at $550^{\circ} \mathrm{C}$ for $18 \mathrm{~h}$ the $\mathrm{FePd}$ alloy is ordered and possesses a tetragonal structure with the space group $P 4 / \mathrm{mmm}$. This is evidenced by the tetragonal splitting of the fundamental reflections (200) and (220) and by the presence of narrow superlattic reflections $(001)$, (110) and (201) in the X-ray diffraction pattern of the FePd alloy. The upsetting of such alloy $(P=3 \mathrm{GPa}$, $n=0)$ results in the disappearance of superlattice reflections in the diffraction patterns [33]. The fundamental reflections undergo a strong asymmetric broadening, just as after deformation of the disordered FePd alloy (Fig. 8). Similar X-ray diffraction patterns were taken earlier for $\mathrm{L}_{0}$ ordered FePd samples after severe (70-90\%) cold-rolling deformation $[11,14]$ : instead of tetragonal doublets, single reflections were observed, as in the common case of an alloy with a fcc lattice. However, the position of these single reflections did not correspond to those for a disordered fcc phase and the ratio $c / a$ determined from the structure reflections was not equal to $1(c / a \neq 1)$. The $\mathrm{X}$-ray analysis, performed with the use of a program [35], showed that as a result of HPT deformation of the ordered FePd alloy a disordered bct phase forms (see interpretation of the experimental diffraction pattern in Ref. [33] and Fig. 8). The volume fraction of the disordered bet phase depends on pressure $P$ and the number of revolutions $n$ at a constant $P$ [33]. Thus, at $P=3 \mathrm{GPa}, n=0$, the FePd sample contains $70 \%$ of the disordered bet phase (A6), which earlier was not observed in such alloys, and 30\% of the disordered fcc phase (A1). At $P=6 \mathrm{GPa}, n=0$, the volume fractions of these phases make up $77 \%$ and $23 \%$, respectively. Additional shear deformation via rotation of the anvils results in the phase transition A6 $\rightarrow$ A1. The change in the relative volumes of the phases in the samples with increasing the number of revolutions at a constant $P=6 \mathrm{GPa}$ is shown in Fig. 10a. It is seen that even after $n=10, \sim 30 \%$ the bct phase is retained in the sample.

Annealing of the sample disordered by means of HPT deformation results in the $\mathrm{A} 1 \rightarrow A 6 \rightarrow \mathrm{L} 1_{0}$ transformation (Fig. 10b). In a correct description of the $\mathrm{A} 6$ and $\mathrm{L} 1_{0}$ phases that possess the space symmetries $14 / \mathrm{mmm}$ and $P 4 / \mathrm{mmm}$, the $c / a$ ratios make up 1.385-1.374 and 1.343, respectively [33]. In the traditional description of the A6 and $\mathrm{L} 1_{0}$ phases as fct structures, which according to Ref. [50] is incorrect, these ratios are $0.98-0.97$ and 0.95 respectively.

\subsection{Modification of scheme of the structural transition $A 1 \rightarrow L 1_{0}$}

In Fig. 11, a scheme of the structural transition $\mathrm{A} 1 \rightarrow \mathrm{L}_{0}(\mathrm{Fm} \overline{3} \mathrm{~m} \rightarrow \mathrm{P} 4 / \mathrm{mmm})$ is shown, which takes into account the above results and remarks of Ref. [50] (compare with Fig. 1). Under the chosen description, the unit cell of the bct phase is turned with respect to the unit cell 


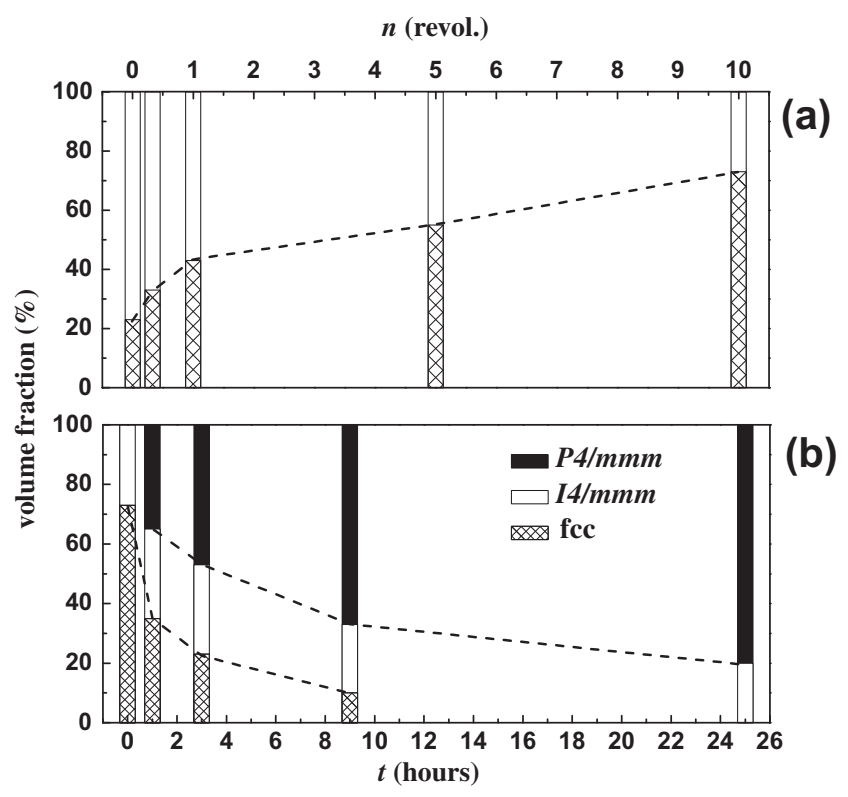

Fig. 10. (a) Dependence of the phase composition of the equiatomic FePd alloy on the number of revolutions upon HPT at $P=6 \mathrm{GPa}$ in the initial ordered state and (b) on the duration of subsequent annealing at $T=450^{\circ} \mathrm{C}$ after HPT at $P=6 \mathrm{GPa}, n=10$.

of the initial fcc phase by an angle of $45^{\circ}$ around the direction [001], so that its tetragonal axis coincides with the [001] axis of the fcc phase, and the axes [010] and [100] are located along the directions [110] and [110] of the fcc phase. (Each of the axes $\langle 100\rangle$ of the fcc lattice can serve as a tetragonal axis of the bet lattice.) Thus, the axes

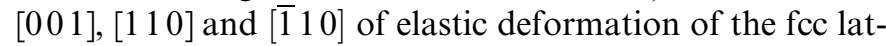
tice (compression along the [001] axis and displacement of atoms located along the directions [110] and [1 10$]$ ) become the main crystallographic axes [001], [0 10$]$ and $[100]$ of the disordered bet phase.

According to the X-ray data [33], the changes in the lattice parameter $\left(a_{\mathrm{fcc}}-c_{\mathrm{bct}}\right) / a_{\mathrm{fcc}}$ along the compression axis and $\left(a_{\mathrm{fcc}}-\sqrt{ } 2 \cdot a_{\mathrm{bct}}\right) / a_{\mathrm{fcc}}$ along the displacement axes are $\sim 2 \%$ and $<1 \%$, respectively. Hence, a dominant contribution to the elastic deformation of the lattice upon the phase transformation $\mathrm{A} 1 \rightarrow \mathrm{A} 6(F m \overline{3} m \rightarrow I 4 / \mathrm{mmm})$ is made by the so-called homogeneous Bain deformation (compression or tension along the [001] axis) $[25,27]$. In the frame of the description chosen, the mutual disposition of the fcc and bct lattices is in agreement with the known Bain orientation relationships for the martensitic transformations fcc $\rightarrow$ bcc (bct): $\left[\begin{array}{lll}0 & 0 & 1\end{array}\right]_{\mathrm{bct}}\left\|\left[\begin{array}{lll}0 & 0 & 1\end{array}\right]_{\mathrm{fcc}},\left[\begin{array}{lll}0 & 1 & 0\end{array}\right]_{\mathrm{bct}}\right\|\left[\begin{array}{lll}1 & 1 & 0\end{array}\right]_{\mathrm{fcc}},\left[\begin{array}{lll}1 & 0 & 0\end{array}\right]_{\mathrm{bct}} \|\left[\begin{array}{lll}1 & 1 & 0\end{array}\right]_{\mathrm{fcc}}$,

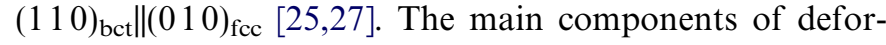
mation upon such transformations are homogeneous Bain deformation, shear deformation along the $\{110\}\langle\overline{1} 10\rangle$ systems and twinning over the planes $\{111\}$ of the initial fcc phase. With the above orientation relationship, the planes $(101)_{\text {bct }} \|(111)_{\text {fcc }}$ actually can serve as the planes of conjugation of two phases.

\section{Conclusion}

The overall results from optical polarization, TEM, thermomagnetic and $\mathrm{X}$-ray studies performed on single (a)
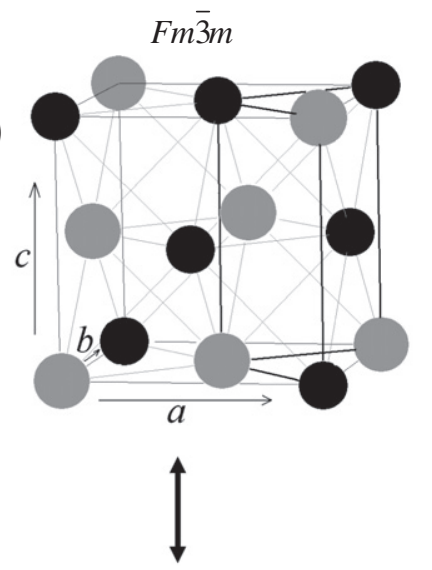

(b)

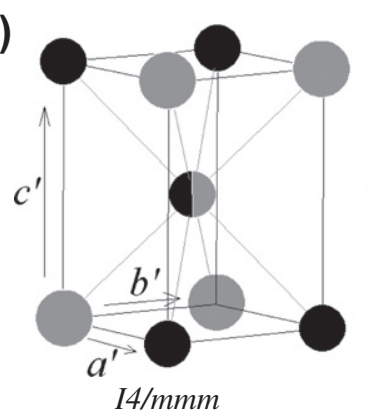

$$
\begin{aligned}
& {[001]_{F m \overline{3} m} \|[001]_{I}} \\
& {[110]_{F m \overline{3} m} \|[010]_{I}} \\
& {[\overline{1} 10]_{F m \overline{3} m} \|[100]_{I}}
\end{aligned}
$$

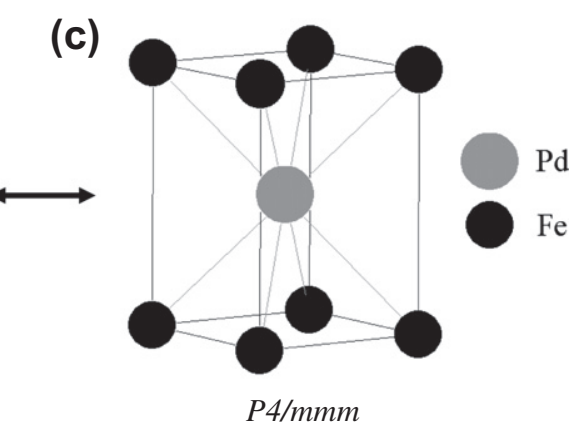

Fig. 11. Scheme of the structural transition $\mathrm{A} 1 \leftrightarrow \mathrm{L} 1_{0}(F m-3 m \leftrightarrow P 4 / m m m)$ through the intermediate phase $\mathrm{A} 6(I 4 / m m m)$. 
crystals and samples of FePd alloy, which had been nanostructured via HPT deformation, testify that upon the $\mathrm{A} 1 \leftrightarrow \mathrm{L} 1_{0}$ transformation a metastable disordered bodycentered tetragonal phase is formed. The atomic ordering proceeds in the tetragonally distorted lattice, and the phase transformation $\mathrm{A} 1 \rightarrow \mathrm{L}_{0}$ is a combination of the phase transitions of different types: cooperative displacement and atomic ordering. The first stage of the $\mathrm{A} 1 \rightarrow \mathrm{L}_{0}$ transformation is a martensitic transition $\mathrm{fcc} \rightarrow$ bct (A1 $\rightarrow \mathrm{A} 6$ ). The second stage is the formation and growth of nuclei of the ordered phase ( $\mathrm{A} 6 \rightarrow \mathrm{L} 1_{0}$ ). According to changes of the relative intensities of the superlattice reflections [7], upon annealing of the quenched FePd alloy a continuous increase of the integral, i.e., averaged over the whole volume, degree of long-range order occurs. However, a thorough analysis of special features in the diffraction patterns evidences that at different stages of annealing, the FePd samples contain discrete regions in which the degrees of long-range order and of tetragonal distortions of the crystal lattice are different [7]. A possible vacancy mechanism of the formation of regions with different degrees of long-range order was suggested and discussed in Ref. [51].

It is necessary to underline that, in accordance with the symmetry theory $[17,18,48]$, only completely ordered structures consisting of layers of different atoms $\mathrm{A}$ and $\mathrm{B}$, which alternate in one of the $\langle 100\rangle$ directions of the initial fcc lattice, belong to the space group $P 4 / \mathrm{mmm}$. This space group is characterized by quite a definite set of symmetry operations. Disordered and partially ordered tetragonal structures with low-order parameters initially have a bct lattice with a set of symmetry operations different from that peculiar to the $\mathrm{L}_{0}(P 4 / \mathrm{mmm})$ structure and must be referred to the space group $I 4 / \mathrm{mm}$ (phase A6). This difference allowed us to identify $\mathrm{A} 6$ and $\mathrm{L}_{0}$ phases in our experiments.

The existence of the bct phase can be considered as a reason for all the structural peculiarities of the $\mathrm{L} 1_{0}$ alloys that were reported in earlier papers [7-14,20,22-24,5255]. Among them one can pick out: (1) the difference in the $c / a$ ratio determined from the fundamental and superlattice reflections [7]; (2) the existence of inequality $c / a \neq 1$ in the case of unsplit reflections (i.e. the absence of the tetragonal doublets) in the X-ray patterns of the CoPt, FePt and FePd alloys after plastic deformation [11,14]; (3) the appearance and disappearance of an additional sextet in the Mossbauer spectra (which could not be related to either initial ordered or disordered state with the cubic lattice) taken after milling of the ordered FePt alloy [52]; (4) the presence of two tetragonal phases with different $c / a$ ratios after heat treatment of the Fe-Pt alloy of a nonstoichiometric composition [53]; (5) the significant difference in the Curie temperatures of the ordered phase measured by different authors [10,23,45-47]; (6) multistep spatial twinning at early stages of the atomic ordering in the $\mathrm{CoPt}$ alloy $[54,55]$ and others.
The conclusion on the formation of intermediate phases upon the so-called reconstructive phase transitions bcc-hcp and bcc-fcc resulted from the theoretical analysis of the transformations in the mode of cooperative thermal oscillations of the atomic planes [56]. In these cases intermediate phases take part in the subgroup relation between the symmetry groups of the phases above and below the transformation point. In this way the Landau conception of the existence of a subgroup relation upon the phase transformation was confirmed. Hence, the formation of intermediate phases is a regular stage of phase transformations of different types.

From our data it follows that the samples of the equiatomic alloy FePd subjected to HPT deformation and subsequent annealing contain a considerable fraction of the bct phase in the states with a maximal coercivity $\left(H_{\mathrm{c}}^{\max } \approx 130 \mathrm{kA} \mathrm{m}^{-1}\right)$. According to the theoretical estimates for the alloy CoPt [4], the magnetic anisotropy constant of the disordered tetragonal phase makes up $20 \%$ of the constant of magnetic crystallographic anisotropy of the ordered $\mathrm{L} 1_{0}$ phase and is opposite in sign. If the same ratio of the anisotropy constants and, correspondingly, of the anisotropy fields holds for the bct and $\mathrm{L} 1_{0}$ phase in the case of FePd, the presence of $\sim 30-50 \%$ bct phase [33] drastically hampers the realization of high values of $H_{\mathrm{c}}^{\mathrm{max}}$. The situation is complicated by a significant inhomogeneity of grains in size $D \approx 50-200 \mathrm{~nm}[33,34]$. Therefore, to achieve higher hysteresis properties in bulk samples of FePd alloy, a further search for new means of governing microstructure and phase composition in the course of the phase transformation $\mathrm{Fm} \overline{3} \mathrm{~m} \rightarrow I 4 / \mathrm{mmm} \rightarrow P 4 / \mathrm{mmm}$ is necessary.

\section{Acknowledgments}

The work was supported by the Program of the Ural Branch of RAS, Project No. 12-П-23-2005 and the Russian Foundation for Basic Research, Project No. 13-02-00198. $\mathrm{X}$-ray analysis and electron microscopy examination of the FePd samples were conducted in the X-ray Diffraction Analysis and Electron Microscopy Centers, Ural Division, Russian Academy of Sciences. The authors are very grateful to V.V. Nikolaev, A.V. Dobromyslov, G.V. Ivanova, X. Sauvage and F.A. Kassan-Ogly for valuable remarks in the course of discussion of some results of the work.

\section{References}

[1] Newkirk JB, Geisler AN, Martin DL, Smoluchowski R. Trans AIME 1950;188:1249.

[2] Van Laar B. J Phys 1964;25:600.

[3] Weller D, Moser A, Folks L, Best ME, Lee W, Toney MF, et al. IEEE Trans Magn 2000;36:10.

[4] Razee SSA, Staunton JB, Ginatempo B, Pinski FJ. Phys Rev B 2001;64:014411-1.

[5] Staunton JB, Ostanin S, Razee SS, Gyorffy B, Szunyogh L, Ginatempo B, et al. J Phys: Condens Matter 2004;16:5623.

[6] Shima H, Oikawa K, Fujita A, Fukamichi K, Ishida K, Sakuma A. Phys Rev B 2004;70:224408-1. 
[7] Ivanova GV, Magat LM. Fiz Met Metalloved 1975;39:999 [in Russian].

[8] Zhang B, Soffa WA. IEEE Trans Magn 1991;27:5328.

[9] Yermakov AYe, Sokolovskaya NI, Tsurin VA, Ivanova GV, Magat LM. Phys Metals Metallogr 1978;46:737.

[10] Yermakov AYe, Maykov VV. Phys Metals Metallogr 1990;69:198.

[11] Ivanova GV, Shchegoleva NN, Magat LM, Shur YaS. Phys Metals Metallogr 1972;34:107.

[12] Deshpande AR, Wiezorek JMK. Acta Mater 2004;52:2903; Deshpande AR, Wiezorek JMK. JMMM 2004;270:157.

[13] Laughlin DE, Srinivasan K, Tanase M, Wang L. Scripta Mater 2005;53:383.

[14] Kulovits AK, Deshpande AR, Wiezorek JMK. Intermetallics 2009;17:865.

[15] Soffa WA, Laughlin DE, Singh N. Solid State Phenom 2011;172174:608.

[16] Aizu K. J Phys Soc Jpn 1969;27:387.

[17] Naish VE, Syromyatnikov VN. Kristallografiya 1976;21:1085.

[18] Sirotin YuI, Shaskol'skaya MP. Fundamentals of crystal physics. Moscow: Nauka; 1979.

[19] Toledano JC, Toledano P. Phys Rev B 1980;21:1139.

[20] Orehotsky J, Orehotsky JL. J Appl Phys 1987;61:4240.

[21] Khachaturyan AG. Theory of structural transformations in solids. New York: Wiley; 1983.

[22] Vlasova NI, Kandaurova GS, Rjabinina OS. Phys Metals Metallogr 2004;98:272.

[23] Vlasova NI, Popov AG, Shchegoleva NN. Phys Metals Metallogr 2009; 107:359.

[24] Vlasova NI, Shchegoleva NN, Popov AG, Kandaurova GS. Phys Metals Metallogr 2010;110:449.

[25] Roitburd AG. Phys-Usp 1974;113:69.

[26] Kassan-Ogly FA, Naish VE, Sagaradze IV. Phase Trans 1994;49:89.

[27] Pushin VG, Kondrat'ev VV, Khachin VN. Pretransition effects and martensitic transformations. Ekaterinburg: Ural. Otd. Ross. Akad. Nauk; 1998 [in Russian].

[28] Shuvalov LA. Izv Akad Nauk SSSR 1979;43:1554 [in Russian].

[29] Barsh GR, Krumhansl JA. Phys Rev Lett 1984;53:1069.

[30] Barsh GR, Krumhansl JA. Metall Trans A 1988;19:761.

[31] Bilby BA, Christian JW. Martensitic transformations. The mechanism of phase transformations in metals. London: The Institute of Metals; 1956.

[32] Valiev RZ, Islamgaliev RK, Alexandrov IV. Prog Mater Sci 2000;45:103.
[33] Vlasova NI, Gaviko VS, Popov AG, Shchegoleva NN, Stashkova LA, Gunderov DV, et al. Solid State Phenom 2011;168-169:392.

[34] Chbihi A, Sauvage X, Genevois C, Blavette D, Gunderov D, Popov AG. Adv Eng Mater 2010;12:708; Chbihi A, Sauvage X, Genevois C, Blavette D, Gunderov D, Popov AG. Solid State Phenom 2011;172-174:703.

[35] Kraus W, Nolze G. J Appl Crystallogr 1996;29:301.

[36] Tyapkin YuD, Pushin VG, Romanova RR, Buinov NN. Fiz Met Metalloved 1976;41:1040 [in Russian].

[37] Oshima R, Sugiyama M, Fujita FE. Metall Trans A 1988;19:803.

[38] Kondrat'ev VV. Fiz Met Metalloved 1979;47:102 [in Russian].

[39] Sokolovskaya NI, Shchegoleva NN, Kandaurova GS. Phys Metals Metallogr 1976;41:42.

[40] Vlasova NI, Kandaurova GS, Lukjanin VV, Maltsev VN, Shchegoleva NN. JMMM 1995;147:198.

[41] Klassen-Neklyudova MV. Mechanical twinning of crystals. New York: Consultant Bureau; 1964.

[42] Marcinkowski MJ. Electron microscopy and strength of crystals. New York: Wiley; 1963.

[43] Kandaurova GS, Beketov VN. Fizika tverd tela 1974;16:1857 [in Russian].

[44] Hubert A, Schäfer R. Magnetic domains: the analysis of magnetic microstructure. New York: Springer; 1998. p. 405.

[45] Kussmann A, Jessen K. Z Metallkde 1963;54(9):504

[46] Wang L, Fan Z, Roy AG, Laughlin DE. J Appl Phys 2004;95(11): 7483.

[47] Lyubina J, Gutfleisch O, Isnard O. J Appl Phys 2009;105(07):A717.

[48] Barrett CS, Massalski TB. Structure of metals. New York: Pergamon Press; 1980.

[49] Jing L, Gauntt BD, Dickey EC. Acta Mater 2010;58:5009.

[50] Naish VE. Phys Metals Metallogr 1999;87:103.

[51] Soffa WA, Puschl W, Pfeiler W. Intermetallics 2003;11:161.

[52] Yermakov AYe, Sorokina TA, Tsurin VA, Lebedev IjG, Philippov BN, Ilijchenko NG, et al. Fiz Met Metalloved 1979;48:1180 [in Russian].

[53] Yermakov AYe, Maikov VV, Teitel' EI, Novikov SI, Andreeva OB, Magat LM, et al. Phys Metals Metallogr 1990;70:28.

[54] Plakhti VD, Tyapkin YuD. Metallophysics 1984;6(4):744.

[55] Vlasova NI, Shchegoleva NN, Kandaurova GS, Shilova NF. Phys Metals Metallogr 2001;91:559.

[56] Kassan-Ogly FA, Arkhipov VE, Shestakov AE. Phys Metals Metallogr 2010;109(6):568. 